\title{
PET/MRI vs PET/CT in Head and Neck imaging: When, Why, and How?
}

\section{Authors:}

Joshua Ryan, MD (Indiana University School of Medicine)

Vasantha Aaron, MD (Indiana University School of Medicine)

Justin Sims, MD (Indiana University School of Medicine)

\section{Corresponding Author:}

Joshua Ryan (ryanjl@iu.edu or jlryanmed@gmail.com)

\section{Present address:}

Indiana University School of Medicine

Department of Radiology and Imaging Sciences

550 N. University Blvd. Room 0663

Indianapolis, IN 46202

Conflicts of Interest: The authors do not have any financial conflicts of interest pertaining to the subject of the article.

Key Words: Squamous Cell Carcinoma; PET/CT; PET/MRI; Oncologic imaging; PET; Imaging protocols

\section{Abstract:}

The increasing availability of hybrid PET/MRI systems has led to a breadth of new publications and opportunities for use of PET/MRI. While PET/CT has been a valuable tool for oncologic staging, including head and neck malignancy, there are several theoretical and practical advantages a PET/MRI system would have over PET/CT in head and neck imaging. This review article discusses the established role of $\mathrm{PET} / \mathrm{CT}$, early evidence for the role of PET/MRI, and protocol considerations for both PET/CT and PET/MRI as they apply to head and neck imaging.

This is the author's manuscript of the article published in final edited form as: 


\section{Introduction:}

Malignancy in the head and neck encompasses a broad array of diagnoses, including mucosal surface (epithelial) malignancies of the sinonasal cavity and aerodigestive tract, primary and secondary salivary gland malignancies, thyroid cancer, skin cancer, orbito-ocular malignancies, skeletal involvement by tumors, and malignancies involving the lymphoid tissues. The most common subtype of head and neck malignancy is mucosal surface squamous cell carcinoma (SCCa), which is the histologic type in greater than $90 \%$ of mucosal surface malignancies, and may arise in the sinonasal mucosa, nasopharynx, oral cavity, oropharynx, hypopharynx, and larynx. ${ }^{1}$ In 2012, a worldwide estimated 300,400 new cases and 145,400 deaths arose from oral cavity cancer alone. ${ }^{2}$ It is estimated that in 2019 , there will be 53,000 new cases of cancer within the oral cavity or pharynx diagnosed in the United States. ${ }^{3}$ Well known risk factors for head and neck cancer included tobacco use and alcohol, which are also known risk factors for other malignancies in the aerodigestive tract (lung and esophagus). ${ }^{1}$ Human Papilloma Virus (HPV) is an increasingly recognized risk factor for head and neck cancer, which has dramatically changed the demographic of head and neck cancer patients and shifted the typical age of onset of head and neck cancer downward. 1,4,5 Of note, due to increasing incidence of HPV associated head and neck malignancy, the American Joint Committee on Cancer (AJCC) now includes a separate category for staging of HPV-related oropharyngeal cancer. ${ }^{4}$

In the primary initial workup of a head and neck malignancy, imaging plays an essential role, and is generally believed to be more useful in advanced tumor stages. ${ }^{4}$ TNM staging developed by the AJCC is subtyped into multiple sites of origin. ${ }^{4}$ Accurate 
staging is important in treatment planning and prognosis. Owing to the complex anatomy of the head and neck region, imaging plays a vital role in supplementing diagnosis, treatment planning, and disease monitoring in head and neck malignancy. ${ }^{6}$ In clinical practice, specific imaging is typically selected on a case by case basis, and usually involves contrast enhanced Neck CT or MRI. For patients who are suspected to have more advanced disease (stage III or IV), FDG PET/CT is a useful tool that provides high sensitivity for distant disease. ${ }^{1,6,7}$

Whole body PET/MR imaging systems became available in $2010 .{ }^{8}$ Since its introduction, hybrid PET/MR has grown in availability and has begun to be adopted for clinical use, although is still not widely available in the medical landscape. One of the primary theoretical advantages of PET/MRI over PET/CT is the improved soft tissue contrast of MRI compared to CT. This is especially advantageous when imaging the complex anatomical structures that reside within the head and neck. ${ }^{8} \mathrm{PET} / \mathrm{MRI}$ also allows for a significant reduction in radiation dose to the patient compared to PET/CT. ${ }^{9}$

This chapter will discuss the current role of 18F-FDG PET/CT in head and neck imaging and contrast the more established role of PET/CT against the emerging technology of hybrid PET/MRI. Additionally, this article will briefly review protocolling for PET/CT and PET/MRI. 


\section{Established role of 18F-FDG PET/CT in head and neck cancer.}

FDG PET/CT is commonly performed in clinical practice for the workup and staging of head and neck malignancies. In many cases, patients have already undergone CT or MRI and endoscopic biopsy prior to undergoing a PET/CT, and an established tissue diagnosis is known. The National Comprehensive Cancer Network (NCCN) guidelines generally recommend FDG PET/CT in workup of stage III or IV disease, or as clinically indicated for most sites of origin. ${ }^{1}$

\section{Primary Tumor}

A recent study of 35 patients by Chaput et al showed greater diagnostic accuracy with higher sensitivity of FDG PET/CT (83\%) compared to MRI (63\%) for detection of T1-T2 category head and neck SCCa, suggesting efficacy of FDG PET/CT in lower stage disease. ${ }^{10}$ When PET is performed with a contrast enhanced CT, diagnostic CT images can be obtained from a single examination and may obviate the need for additional imaging for staging (CT or MR). ${ }^{11}$ Of note, at our institution, all PET/CT exams are performed with iodinated IV contrast unless there are medical contraindications for the patient (contrast allergy or renal insufficiency). PET/CT protocols are discussed in detail later in this chapter

\section{Nodes and Metastases}

FDG PET/CT has also been established as the optimal imaging study for staging of nodal metastases and distant metastatic disease, backed by recommendations from the AJCC and NCCN for evaluation in stage III and IV disease (Figure 1). ${ }^{1,4}$ A meta- 
analysis by Sun et al. showed greater pooled per neck level sensitivity of FDG PET CT (84\%) for detection of regional nodal metastases over CT or MR (63\%). ${ }^{12}$ In addition, a recent prospective cohort study by Rohde et al. demonstrated superiority of PET/CT for detection rates of distant metastases and second primary malignancy over the combination of radiographs, CT, and MRI based on geographical preferences of Europe and US. ${ }^{13}$

\section{Cancer of Unknown primary}

The use of 18F-FDG PET/CT is also recommended for diagnoses beyond the workup of a known primary site of malignancy within the head and neck. Notably, PET/CT has a role in helping identify the primary site in cancer of unknown primary, which is typically discovered as enlarged cervical lymph nodes with pathology of squamous cell carcinoma, adenocarcinoma or undifferentiated epithelial tumors (Figure 2). ${ }^{1}$ A meta-analysis by Zhu et al. noted that $18 \mathrm{~F}-\mathrm{FDG}$ PET/CT had an overall detection rate of 0.44 , pooled sensitivity of 0.97 , and specificity of 0.68 for primary site detection in an analysis of 7 studies.

\section{Mucosal Melanoma}

18F-FDG PET/CT should also be considered for staging and screening for metastatic disease in the setting of mucosal melanoma, which is generally seen in an older population with median age at diagnosis $70 .{ }^{4,15}$ Mucosal melanoma is a rare entity, encompassing only $1.3 \%$ of known melanoma cases, with about $55 \%$ occurring in the head and neck region. ${ }^{16}$ Most of the head and neck mucosal melanoma cases arise in the paranasal sinuses and nasal cavity. ${ }^{4}$ Unfortunately, mucosal melanoma is 
an aggressive entity, and most patients progress to metastatic disease. ${ }^{17}$ Metastases can occur most commonly in lung, bone, peritoneum, and central nervous system; thus $\mathrm{PET} / \mathrm{CT}$ is an ideal imaging modality to evaluate for distant metastases. ${ }^{17} \mathrm{MRI}$ Brain is also usually indicated for evaluation of brain metastases in melanoma patients.

\section{Salivary gland malignancy}

According to the AJCC, there is no established role for PET/CT for the evaluation of primary salivary gland malignancy, with CT and MR potentially providing a complementary role in staging and diagnosing major salivary gland tumors. ${ }^{4} \mathrm{~A}$ recent prospective study by Park et al. showed 18F-FDG PET/CT to be useful in the detection of metastases and additional primary cancers in these patients, along with a predictive role in survival outcomes. ${ }^{18}$ This study suggests that expansion of the current role of 18F-FDG PET/CT in primary salivary gland cancer may be an area of further research.

\section{Treatment evaluation and post-treatment FDG PET}

In the post-treatment setting following radiation (RT) alone or chemotherapy and radiation (CRT), NCCN guidelines state that 18F-FDG PET should be considered after therapy in patients who exhibit clinical response to treatment with a wait time of at least 12 weeks to minimize post-treatment uptake and false positive results (Figure 3 ). ${ }^{1} \mathrm{~A}$ meta-Analysis performed by Gupta et al. showed excellent sensitivity (79.9\%), specificity (87.5\%) and negative predictive value (NPV; $95.1 \%)$, but a relatively low positive predictive value (PPV; $58.6 \%$ ). ${ }^{19}$ This meta-analysis suggests that PET/CT is a more accurate modality for informing clinical decision making when the results are 
negative. ${ }^{19}$ While it has generally been established that post-therapy $18 \mathrm{~F}-\mathrm{FDG}$ PET/CT should occur around 12 weeks or greater post therapy, the NCCN suggests that $18 \mathrm{~F}$ FDG PET/CT can also be considered earlier in patients who have evidence of persistent or progressive disease. ${ }^{1}$ However, there is currently no well-established role of $18 \mathrm{~F}$ FDG PET/CT in assessing early response while undergoing treatment. ${ }^{20}$

The recently published PET-NECK randomized controlled trial performed by Mehanna et al. showed non-inferiority of 18F-FDG PET/CT surveillance after CRT in patients with advanced nodal (N2 or N3) disease compared to patients who underwent radical neck dissection. ${ }^{21}$ The results of the PET-NECK study show a viable noninvasive alternative for disease surveillance in patients with treated metastases.

Another recently published multicenter trial by Lowe et al, the ACRIN 6685 trial, showed a $18 \mathrm{~F}-\mathrm{FDG}$ PET/CT to have a high NPV (87\%) for a clinically N0 neck in patients with squamous cell carcinoma. ${ }^{22}$ This study also noted that surgical planning was changed in approximately $21 \%$ of patients after surgeon review of the PET/CT. The findings from this study demonstrate the value of $18 \mathrm{~F}-\mathrm{FDG}$ PET/CT in determining treatment course for clinically NO necks.

\section{Prognostic value of 18 F-FDG PET/CT}

There has also been a focus in the recent literature on the prognostic value of $18 \mathrm{~F}-\mathrm{FDG}$ PET/CT in patients with head and neck malignancy. The most well-known and widely reported semi-quantitative measurement parameter in PET is the maximum standardized uptake value (SUV) within a volume of interest. SUV has been established as a prognostic indicator in head and neck squamous cell carcinoma, with 
increased SUV of primary tumors being a poor prognostic factor. ${ }^{23}$ However a recently published meta-analysis by Wang et al. demonstrated superior prognostic predictive capabilities of metabolic tumor volume (MTV) and total lesion glycolysis (TLG) compared to SUV max. ${ }^{24}$ It should be noted that TLG and MTV are not yet widely used, possibly because they are difficult to perform and require specialized software. Regardless of the measurement parameter employed, 18F-FDG PET/CT has established itself as an important prognostic tool for clinicians. 18F-FDG PET/CT has also shown potential value in the radiation therapy treatment planning, as treatments such as intensity-modulated radiation therapy (IMRT) depend on accuracy of tumor size. ${ }^{20}$

18F-FDG PET/CT is a versatile imaging modality that can help guide clinical decision making in a variety of settings. However, there are known advantages MRI has over CT in head and neck imaging, including better soft tissue contrast and spatial resolution, and lack of ionizing radiation. Combined with the fact that many patients with head and neck malignancy undergo both, these limitations provide a framework for the clinical use of PET/MRI in many head and neck settings.

\section{$\mathrm{PET} / \mathrm{MRI}$ in imaging of head and neck}

\section{Retrospective fusion}

The earliest studies that examined the feasibility of PET combined with MRI in head and neck imaging were fusion studies between separately performed MRI and PET. Nakamoto et al. published a retrospective fusion study in 2009 that showed the 
benefit of PET fusion with MRI in post-treatment patients suspected of having disease recurrence, though there was minimal benefit in initial staging. ${ }^{25}$ Retrospective fusion study by Huang et al. showed that fused PET/MR provided greater performance than $\mathrm{PET} / \mathrm{CT}$, standalone $\mathrm{CT}$, or standalone $\mathrm{MRI}$ in detecting tumor size and local invasion in advanced buccal squamous cell carcinoma. ${ }^{26}$ Kanda et al. performed a retrospective fusion study in 30 patients with oral cavity or hypopharyngeal cancer, which showed greater accuracy of T category assessment with fused PET/MRI and MRI compared to $\mathrm{PET} / \mathrm{CT}$, and greater accuracy of $\mathrm{N}$ category assessment with fused PET/MRI and PET/CT over MRI alone. ${ }^{27}$

\section{Initial experiences with hybrid PET/MRI.}

Boss et al. were the first to publish a study demonstrating the feasibility of a simultaneous PET/MRI system in a study of 8 patients. ${ }^{28}$ Their results showed no image degradation of the accompanying MR images, with the added benefit better spatial resolution of PET images performed with MRI as compared to the PET/CT, due to the smaller diameter of PET detector ring and smaller scintillation crystal sizes on their PET/MRI system. ${ }^{28}$ These initial results were supported by the findings of Platzek et al., who showed no MRI artifacts in PET images in a 20-person prospective study in patients with head and neck squamous cell carcinoma. ${ }^{29}$ Another early study showed a good qualitative comparison between PET/MRI and PET/CT images and a correlation between SUVs between the PET/MRI and PET/CT. ${ }^{30}$ Along with correlating SUVs between PET/MRI and PET/CT, Covello et al. demonstrated the multiparametric abilities of PET/MRI in the evaluation of head and neck imaging (SUVs, diffusion, and perfusion parameters). ${ }^{31}$ 


\section{PET/MRI vs PET/CT and conventional imaging; The evidence}

\section{TNM staging}

Following the feasibility studies, the next step in research of PET/MRI for image quality in the head and neck was to compare PET/MRI to PET/CT and to conventional imaging (MRI and CT). Several studies have shown the non-inferiority of PET/MRI in staging these malignancies. For example, a prospective study by Kuhn et al, evaluated 150 patients with head and neck cancer presenting for a PET/CT evaluation. Using a trimodality PET/CT-MR setup, their results showed that contrast enhanced PET/MR performed significantly better than contrast enhanced PET/CT in evaluation of primary tumors; however, there was no significant difference in evaluation of lymph nodes. ${ }^{32}$ Another study by Platzek et al. noted that there was no significant difference between PET/MR and MRI and PET alone in the accuracy of lymph node staging in 38 patients. ${ }^{33}$ A small study of 17 patients by Kubiessa et al. showed no statistically significant difference in diagnostic performance (Sensitivity, Specificity, PPV and NPV) between PET/CT and PET/MRI in evaluation of malignant findings in patients with head and neck cancer. ${ }^{34}$ Partovi. et al also found comparable results between PET/CT and PET/MRI for detection of yymph nodes and metastases in their prospective study of 14 patients with head and neck cancer (figure 4). ${ }^{35}$

A small study of 16 patients with laryngeal cancer by Cavaliere et al. showed that PET/MRI is useful for the primary staging of laryngeal cancer and is comparable to PET/CT. ${ }^{36}$ Their study showed a high correlation between imaging findings and endoscopic/histological evaluation for PET/MRI. They also reference the ability of MRI in determining cartilage invasion in suggesting potential for PET/MRI in the evaluation of 
laryngeal cancer. ${ }^{36,37}$ Cavaliere et al. also discuss the advantages of being able to collect metabolic, anatomic and functional parameters in a single imaging study. ${ }^{36}$

Stronger evidence for the use of PET/MRI over PET/CT is seen in a recently published prospective study of 113 patients by Chan et al., which recommended the use of whole-body PET/MRI as a single imaging modality in the staging of nasopharyngeal carcinoma. In this study, PET/MRI outperformed PET/CT and head and neck MRI for T category assessment and showed higher PPV for assessment of distant metastatic disease. ${ }^{38}$

\section{Follow-up imaging and disease recurrence}

The theoretical advantage of PET/MRI over PET/CT may be greatest in the posttreatment setting, as the superior soft tissue contrast resolution of MRI may provide the clinician and radiologist more evidence to determine whether FDG uptake is true recurrence, physiologic activity, or expected post-treatment related uptake. To this effect, Queiroz et al. suggest that PET/MRI may be superior to PET/CT in evaluating unclear FDG findings in follow-up patients with head and neck cancer, but their study showed no significant difference in diagnostic accuracy between PET/CT and PET/MRI. ${ }^{39} \mathrm{~A}$ retrospective analysis by Schaarschmidt et al. showed no significant difference in correct classification of incidental radiotracer uptake in the head and neck on PET/MRI compared to PET/CT in 81 oncologic patients. ${ }^{40}$ In a separate publication, Schaarschmidt et al. also noted no significant difference between PET/MRI, PET/CT, and MRI in local tumor staging or cancer recurrence in a small study of 25 patients. ${ }^{41}$ 
In a study demonstrating the multiparametric capabilities of PET/MRI, Becker et al. showed excellent results for detection of recurrent disease and tumor classification of PET/MRI performed with diffusion weighted imaging (DWI) in the post-treatment setting. ${ }^{42}$ This study notes the value of concordant imaging results between PET, MRI, and DWI in predicting tumor recurrence, and discordant results corresponding to benign post-treatment changes. ${ }^{42}$ These results differ from an earlier study by Queiroz et al., which concluded that DWI was not helpful in the evaluation of head and neck malignancy with PET/MRI. ${ }^{43}$ They also highlight the findings of Rasmussen et al. that 18F-FDG uptake and DWI have independent but complementary roles in head and neck imaging. ${ }^{44}$ Based on the preliminary research, further studies will be necessary to define the role of multiparametric imaging as part of a PET/MRI examination in the head and neck.

\section{Cancer of unknown primary and non-squamous cell carcinoma of the head and} neck.

18F-FDG PET/CT has been firmly established as a valuable tool in the work-up of cancer of unknown primary manifesting as cervical lymph node metastases, and PET/MRI hold promise in this setting, as well. ${ }^{1,14}$ In a study of 20 patients, PET/CT and PET/MRI equally identified $11 / 20$ primary cancer sites. ${ }^{45}$ They also noted that PET/MRI had superior assessment of cervical lesions on PET/MRI and superior detection of pulmonary metastases on PET/CT, although no statistical significance was determined. ${ }^{45}$ To date, no studies have been performed that specifically assess the utility of PET/MRI in salivary gland malignancies or mucosal melanoma. This may be in part due 
to the non-established role in of FDG-PET/CT in assessing salivary gland malignancies, and the rarity of mucosal melanoma.

\section{Specific situations where MRI is preferred over CT.}

There are situations in which the superiority of MRI over CT can be used to infer that PET/MRI may be a preferred modality to PET/CT. One is the evaluation of perineural spread, where MRI is preferred over CT because of its superior soft tissue contrast resolution. ${ }^{46}$ (Figure 5) In reference to PET/MRI, a prospective study by Sekine et al., which used a trimodality PET/CT+MRI system, showed that PET/MRI detected 6 cases of perineural spread, while PET/CT detected only 5. ${ }^{47}$

$\mathrm{MRI}$ is also the imaging modality of choice in the evaluation of salivary gland malignancy and helps with accurate localization and locoregional extension of salivary gland tumors, as well as detection of perineural spread. ${ }^{48} \mathrm{MRI}$ is also generally regarded as superior to CT in evaluation of orbital disease, due to superior contrast resolution and ability to assess extent of disease involving the orbital compartment, perineural spread, and intracranial extension. ${ }^{49}$ Evaluation of subtle skull base invasion and marrow replacement, along with intracranial extension is also better appreciated by MRI, which is currently the gold standard for evaluation of nasopharyngeal carcinoma. ${ }^{38} \mathrm{MRI}$ is also the preferred modality when imaging the sella and parasellar region. ${ }^{50}$ Specifically, the superior soft tissue contrast resolution would be better for staging in evaluating for carotid encasement and cavernous sinus extension. 
Another clinical situation is evaluating the oral cavity and oropharynx in patients with dental hardware. Streak artifact from CT can limit the diagnostic confidence and lesion conspicuity, while MRI can also have signal void due to metallic artifact, it is generally less of a hinderance in diagnostic confidence than CT artifact. (Figure 6).

\section{Summary of findings and suggestions for clinical use of PET/MRI}

The first portion of this chapter has briefly reviewed the evidence for the use of both 18F-FGD PET/CT and PET/MRI in the setting of head and neck cancer. As described, 18F-FDG PET/CT plays a significant and versatile role in head and neck imaging, and its recommendation by the AJCC and NCCN in multiple settings further emphasizes its established role in medicalimaging. However, while the data comparing PET/CT to PET/MRI are limited in number of studies and patients per study, the preliminary results show that PET/MRI is comparable to PET/CT in multiple settings. It is therefore reasonable that PET/MRI should be considered for use in individually selected cases where the benefit of MRI is perceived to be of greater than CT.

Specific examples were PET/MRI could be considered include patients with significant dental artifact that hinders the diagnostic confidence of CT. PET/MRI should also be considered in patients who have suspected perineural disease or have primary tumors that are at high risk for perineural tumor spread, such as salivary gland neoplasms, orbital tumors, and skin cancer. In patients with head and neck malignancy who would be deemed to require a separate MRI and PET/CT for staging or restaging, PET/MRI can provide the convenience of a "one stop shop" and should be considered. 
PET/MRI should also be considered in evaluation of locally advanced tumors where differentiation between tumor and adjacent structures would have an impact on clinical or surgical decision making, including staging of disease. PET/MRI should also be considered for primary tumors near the skull base, orbits, oral cavity, or in primary nasopharyngeal carcinoma. At our institution, the selection of head and neck cancer patients for PET/MRI is typically done in a multidisciplinary approach with multidisciplinary tumor boards providing an ideal platform for physician-to-physician discussions about the capabilities of PET/MRI and the appropriate selection of patients for PET/MRI.

\section{PET/CT protocol considerations}

\section{Background}

PET/CT imaging protocols vary greatly, and imaging of head and neck malignancies highlights some of the differences. In this section, we will review the PET and CT parameters that can be manipulated in order to optimize head and neck scans. More general PET imaging questions, such as appropriate blood glucose levels or $18 \mathrm{~F}$ FDG dosing, will not be discussed.

\section{CT parameters}

The most fundamental question in protocoling the CT portion of a PET/CT study is determining whether the CT should be "diagnostic" or performed only for anatomic localization and attenuation correction. The latter "low dose CT" protocol is the most common approach in the United States with some predominantly academic institutions 
favoring the former. Some institutions perform a combination of both low dose and diagnostic CTs. For example, performance of a low dose CT from the skull to the thigh may be followed by a dedicated, diagnostic, breath-hold chest CT in patients with lung cancer.

Localization and attenuation correction protocols employ low mAs (30-80) and varying $k V p$ settings and may reduce radiation dose by as much as $50-80 \% 51$, however, they come at the cost of increased noise and artifact and do not typically use iodinated contrast. This means that there is little soft tissue contrast and minimal information regarding tumor characterization or extent of local spread can be gleaned from such studies.

The exact definition of diagnostic CT may differ among radiologists; however, adequate spatial resolution with acceptable signal to noise ratio, increased soft tissue contrast, and enough radiation dose to allow for diagnostic characterization of pathologic processes are necessary to consider a study diagnostic. ${ }^{52}$ This requires higher mAs, which is ideally modulated based on the patient topogram, and in patients without contraindications, iodinated contrast administration. The advantages of contrastenhanced CT (CECT) over non-contrast CT (NECT) in evaluating many pathologic processes are well established and include increased lesion conspicuity, lesion characterization, and diagnostic confidence. However, there are very few clinical studies evaluating its benefits in the setting of 18F-FDG PET/CT and no study specifically addresses this question in the setting of head and neck malignancies. There are a few studies of other tumor sites, which have shown the benefits of using CECT with PET imaging. A small retrospective study comparing CECT versus low dose, NECT PET/CT 
whole body imaging in patients with different tumor types showed that CECT added value to a majority of scans. ${ }^{53}$ The greatest benefit in this study was in exact localization of abnormal 18F-FDG uptake on the PET and precise delineation of tumor, which led to a change in management in $21 \%$ of patients. The same group confirmed that CECT PET/CT changed the delineation of primary tumor in $63 \%$ of patients with non-small cell lung cancer compared to NECT PET/CT imaging, and in $20 \%$ of patients the CECT led to the detection of other important clinical findings. ${ }^{54}$ Another small retrospective study showed that CECT PET/CT more accurately identified nodal metastatic spread of rectal cancer over NECT PET/CT. 55

To date, only two studies has been published comparing CECT and NECT PET/CT in head and neck malignancies, however, neither study was designed to evaluate the benefits specific to CECT. One study was performed to determine whether the use of CECT would change interpretation of the PET portion of the scan compared to low dose NECT PET/CT due to potential differences in attenuation correction. 56 While the study authors concluded that there was no clinically significant difference in interpretation of the PET when using CECT or NECT for attenuation correction, the study did not address changes in interpretation or management based on the CTs themselves. The second study compared whole body NECT PET/CT to dedicated head/neck CECT PET/CT. ${ }^{57}$ The dedicated head/neck CECT PET/CT was performed after longer equilibration compared to the whole body NECT PET/CT. In addition, the PET was obtained over a longer time period in the dedicated protocol and other PET acquisition and reconstruction parameters were altered as well. This study design confounded the ability to determine whether the CECT itself led to improved detection of 
nodal metastases or NECT when performed with PET/CT. Regardless, the study did determine that dedicated head/neck CECT PET/CT identified more cervical nodal metastases compared to whole body NECT PET/CT and suggests that optimizing both PET and CT protocols may significantly alter interpretation and patient management.

We do know that identifying and defining perineural spread, cartilage involvement, necrosis, and nodal extracapsular extension of tumor is crucial in the staging and treatment planning of patients with these malignancies and may only be delineated with diagnostic contrast-enhanced CT or MRI. CECT performed with PET may yield all the necessary information and negate the need for additional, separately performed contrast-enhanced CT or MRI, which lead to additional cost and medical visits for the patient. Another reason to consider CECT over NECT PET/CT in head and neck malignancies is the potential for avoiding false negative interpretations. Cystic and mucinous tumors or cystic nodal metastases may show little uptake of 18F-FDG and may not be identified or characterized as abnormal based upon the PET data alone (Figure 7). Finally, the ability to reconstruct the diagnostic CT into sagittal and coronal views, which are standard series for all head/neck imaging at our institution and review the images as fused and unfused data sets gives additional, useful information to interpreting physicians.

There are pros and cons to both low dose NECT and diagnostic CECT PET/CT protocols. The major benefits to performing low dose NECT with PET include lower radiation dose, limited pre-scan patient evaluation, minimal CT training for technologists, and no need for physician presence during scans. As mentioned previously, CT images are noisy and show increased artifact without additional 
information beyond basic localization of tumor sites. Diagnostic CT protocols employing intravenous contrast require fully trained technologists in both PET and CT, pre-scan evaluation of renal function and contrast allergies, physician presence in case of allergic reaction, and increased radiation dose to the patient. In addition, if iodinated contrast is administered, interpreting physicians must be aware of possible pitfalls to interpretation. While there is no clinically significant change in SUVs when CECT is used for attenuation correction of the PET data, artifacts can arise due to large collections of contrast. ${ }^{58,59}$ For example, contrast pooling within a vein may lead to overcorrection of the PET data and what appears to be focal accumulation of the radiopharmaceutical. Careful evaluation of the underlying CT images and review of the non- attenuation corrected PET data are required to determine whether the "hot" spot is a true pathologic lesion or artifact (Figure 8).

\section{PET parameters}

There is also flexibility in protocoling the PET portion of PET/CTs for head and neck malignancies. The simplest protocol includes a CECT or NECT skull to thigh CT followed by a skull to thigh PET. In this protocol, there is no separate, dedicated neck imaging. This protocol is fast, minimizes the possibility of patient motion, and potentially exposes patients to the least amount of radiation. In head and neck malignancies, dedicated head and neck PET images commonly are obtained at a delayed time point after performance of the skull to thigh PET/CT. Several studies have demonstrated increased sensitivity for detection of small nodal metastases with these types of protocols, however the equilibration time after 18F-FDG injection varies widely from 60 
to 150 minutes (Figure 9). ${ }^{57,60,61}$ At least one study has shown that dedicated, delayed neck PET/CT can lead to decreased specificity in detection of lymph node metastases, presumably because reactive nodes demonstrate abnormal uptake. ${ }^{62}$

Most dedicated head and neck PET acquisitions include longer bed times with reconstructed smaller field of view and higher matrix/decreased pixel size compared to the whole-body PET scan. For example, a dedicated neck PET protocol may include 68 minutes/bed with a $30 \mathrm{~cm}$ field of view and $256 \times 256$ matrix, while the skull-thigh PET protocol may employ 2-4 minutes/bed with a $50 \mathrm{~cm}$ field of view and $128 \times 128$ matrix. The higher number of counts obtained with the longer bed time in the dedicated PET should compensate for the smaller pixels and potential for increased noise. In addition, decreasing the size of the field of view without changing the reconstruction matrix or even increasing it improves spatial resolution. Slice thickness and amount of zoom should be matched with the dedicated CT performed with the PET as well.

If a dedicated neck PET/CT is performed separate from the skull to thigh images, established protocols include performance of a skull to thigh low dose, NECT PET/CT followed by the dedicated neck PET/CT, which may also be performed as a low dose, NECT PET/CT or as a diagnostic, CECT PET/CT. Performing the dedicated PET/CT after the skull to thigh allows for longer equilibration time. Alternatively, the dedicated neck PET/CT/may be performed after a clavicle to thigh PET/CT or vice versa. In this protocol, the patient's arms are down for the neck portion of the study, which limits beam hardening and streak artifact on the CT and, therefore, subsequent artifact on the attenuation corrected PET images. While the technologist is setting-up the clavicle to thigh portion of the study, the patient is instructed to raise the arms above the head, 
which then limits artifact upon scanning of the torso. In addition to producing images with significantly less artifact, this protocol decreases radiation dose to the neck, which is included in only one CT scan. Finally, parameters such as field of view and reconstruction matrix can be optimized for the dedicated neck PET/CT and adjusted for the clavicle to thigh PET/CT, which may require different parameters such as a larger field of view.

\section{PET/CT Protocol with Diagnostic CECT}

There are many ways to optimize both the PET and the CT protocols in the imaging of head and neck malignancies. At our institution, we perform clavicle to thigh PET/CT without contrast followed by dedicated orbit to aortic arch, CECT PET/CT of the neck, which closely mirrors the parameters of standalone diagnostic neck CTs. For example, the soft tissue neck CT protocol at our institution on a 256 slice Philips iCT scanner includes scanning at $120 \mathrm{kVp}, 250 \mathrm{mAs}, 0.993$ pitch, and $0.75 \mathrm{~s}$ rotation time with $2 \mathrm{~mm}$ slice thickness at $2 \mathrm{~mm}$ intervals 70 seconds after the intravenous administration of contrast with axial, sagittal, and coronal reconstructions. This protocol is replicated as closely as possible in our dedicated CECT neck PET/CT.

There are advantages and disadvantages to varying CT and PET parameters in patients with head and neck malignancies, and each patient's unique circumstances should be considered in determining the optimal protocol. 


\section{PET/MRI protocol considerations}

\section{Background}

Hybrid imaging with PET/MR presents unique protocol challenges not present in either standalone imaging technique. One of the initial technical challenges to be overcome for clinical PET/MRI was the need for attenuation correction of the PET images. PET imaging is uniquely affected by the effects of attenuation, resulting in images with dramatically fewer counts in the middle of the imaged object relative to the periphery. With hybrid PET/CT, the CT can serve as a quickly acquired, accurate attenuation (u) map used for attenuation correction of the images. When CT based attenuation correction is utilized, attenuation values of tissues are derived from their measured CT numbers, corrected for the expected attenuation values of $511 \mathrm{KeV}$ photons. ${ }^{63}$ The CT-based method of attenuation correction has superseded older emission-based methods that used radioactive sources to generate attenuation maps due to the ubiquity of PET/CT and the significant lengthening of imaging times created by emission methods.

MRI does not provide a similar analogous source of attenuation correction due to the fact that MR signal is derived from tissue hydrogen density and relaxation properties rather than the attenuation of x-rays. ${ }^{64}$ For a set of MR images to serve for attenuation correction of the PET data, segmentation maps must be generated, most commonly from a DIXON sequence. This sequence is used to segment tissues into air, lung, fat, and soft tissue. Linear attenuation maps based on the expected attenuation of $511 \mathrm{keV}$ photons in the segmented tissues can then be used to perform attenuation correction of the PET images (figure 10). A fifth tissue-bone—can be added to the attenuation 
correction algorithm based on atlas data. This helps to avoid the quantitative errors introduced when the attenuation effects of bone are ignored, however, errors can still occur when an individual patient's bone anatomy deviates significantly from atlas data. ${ }^{65}$ In addition, errors can be introduced due to incorrect tissue segmentation at air tissue interfaces-for example in the paranasal sinuses (figure 11). ${ }^{66}$ There is continued disagreement among experts regarding whether the quantitative errors introduced by MR based attenuation are clinically significant. ${ }^{67}$

The additional sequences required for accurate attenuation correction of the images and the time-consuming nature of MRI relative to CT place a critical emphasis on the need to develop an efficient set of PET/MRI protocols. It is therefore necessary to be judicious with the sequences selected for imaging.

\section{PET/MRI protocol structure and oncologic protocols in the head and neck}

A general PET/MRI protocol structure is as follows. After an equilibration period (typically at least 45 minutes), the patient lies in the PET/MRI gantry (Figure 12). Once localizer MRI sequences are obtained, PET and MRI data are obtained concurrently in sequential PET "beds." While PET data are acquired-typically 2 to 5 minutes of PET data at each bed-basic MRI sequences are obtained in the anatomic regions of each PET bed. These consist of localizer, attenuation correction (AC) MR sequences (typically DIXON), and a wide field of view sequence designed to depict the relevant anatomy (we use coronal T2 weighted single shot fast spin echo images). These MRI sequences are designed to fit within the time frame of PET imaging, and typically occur over a time frame of 15 to 20 minutes. Once the whole-body PET/MR imaging has been completed, dedicated MRI of the primary region of interest is acquired with the imaging 
protocol tailored to help answer the current clinical question, usually with a slightly abbreviated list of sequences to prevent an excessively long duration of imaging. During this period, an additional longer PET acquisition is acquired, resulting in a set of PET images with higher image quality made possible by the longer duration of imaging.

A typical head and neck oncologic PET/MR protocol at our institution begins with skull to thigh PET/MR imaging beginning at the top of the skull and proceeding to the mid thighs. This is then followed by a dedicated neck or brain MRI tailored to answer the relevant clinical questions (e.g., presence of perineural tumor spread, intracranial extension of tumor, etc). For example, a neck PET/MRI (Table 1) to evaluate a malignancy of the suprahyoid neck would consist of axial and coronal T1 non-fat saturation fast spin echo (T1 non-FS FSE) and T2 fat saturation fast spin echo (T2 FS FSE) images. This would be followed by diffusion weighted imaging in the area of clinical concern. After gadolinium administration, a spoiled three-dimensional gradient echo sequence (VIBE on Siemens scanners, LAVA on GE) is obtained, and axial and coronal images are generated. PET acquisition continues throughout most of the duration of the neck sequences. This is then followed by axial breath-hold spoiled gradient echo sequences at mid inspiration at each of the PET bed regions. We have found that these sequences only minimally lengthen the examination and facilitate comparison with previous and future PET/CT imaging in the axial plane.

A facial oncologic PET/MR protocol (Table 2) consists of the above sequences centered on the facial region, though a three-dimensional FLAIR sequence of the brain may be added if there is concern for intracranial involvement. A general orbital PET/MRI protocol consists of axial and coronal T1 FSE, coronal T2 FSE, three-dimensional T2 
SPACE of the orbits, and axial and coronal gadolinium enhanced T1 FS FSE sequences. An oncologic brain protocol (Table 3) would consist of 3D T1 high resolution spoiled gradient echo sequence (MPRAGE on Siemens scanners, SPGR on GE scanners), Axial FLAIR, Axial T2 FSE FS, Axial DWI, and contrast-enhanced 3D MPRAGE or SPGR sequences. I

It should be noted that at our institution, all head and neck PET/MRIs are protocolled with input of both nuclear medicine and head and neck radiologists, who contribute expertise to a patient-specific protocol that is geared toward answering specific staging-related questions. After completion of imaging, this collaborative approach continues with joint read outs of cases by both neuroradiologists and nuclear radiologists. An example case is depicted in Figures 13 through 15. This case study of PET/MR imaging in the initial staging of marginal zone lymphoma highlights the complementary nature of information derived from PET and MRI imaging. The joint interpretation of this case resulted in the correct identification of perineural tumor spread by the interpreting neuroradiologist as well as the identification of widespread areas of intramuscular and subcutaneous lymphomatous infiltration by the nuclear radiologist.

In summary, a clinical PET/MRI protocols begins with whole body PET/MRI imaging consisting of abbreviated MRI sequences designed to allow attenuation correction and provide basic anatomic information in a similar amount of time as clinical PET/CT. This is followed by additional MRI sequences that are designed to answer the clinical question in the area of interest. This strategy typically allows a clinical PET/MRI to be completed in under an hour, allowing the radiologist to answer the question at hand in a time frame acceptable for clinical imaging. 


\section{Conclusion:}

PET/CT is an established modality in oncologic imaging and is widely used in head and neck imaging. The increasing availability of PET/MRI in the medical landscape raises the question about which modality would be most useful in head and neck oncologic imaging. The early literature for PET/MRI largely shows non-inferiority when compared to PET/CT in head and neck imaging, and some studies show advantages of PET/MRI over PET/CT in certain situations, such as the evaluation of nasopharyngeal carcinoma. PET/CT and PET/MRI have separate and unique protocol considerations in regard to head and neck imaging.

\section{References:}

1. National Comprehensive Cancer Network. Clinical Practice Guidelines in Onology: Head and Neck Cancers. https://www.nccn.org/professionals/physician gls/pdf/head-and-neck.pdf. Published 2018. Accessed February 19, 2019.

2. Torre LA, Bray F, SiegelRL, Ferlay J, Lortet-Tieulent J, Jemal A. Global cancer statistics, 2012. CA Cancer J Clin. 2015;65(2):87-108.

3. American Cancer Society. ACS cancer-facts-and-figures. https://www.cancer.org/research/cancer-facts-statistics/all-cancer-factsfigures/cancer-facts-figures-2019.html. Published 2019. Accessed 2/19/2019.

4. AJCC Cancer staging manual. Eighth Edition. Chicago IL :: American Joint Committee on Cancer, Springer; 2017. Amin MB, Edge, S., Greene, F. et al, ed.

5. Rettig EM, D'Souza G. Epidemiology of head and neck cancer. Surg Oncol Clin N Am. 2015;24(3):379-396.

6. Seeburg DP, Baer AH, Aygun N. Imaging of Patients with Head and Neck Cancer: From Staging to Surveillance. Oral Maxillofac Surg Clin North Am. 2018;30(4):421-433.

7. Goel R, Moore W, Sumer B, Khan S, Sher D, Subramaniam RM. Clinical Practice in PET/CT for the Management of Head and Neck Squamous Cell Cancer. AJR Am J Roentgenol. 2017;209(2):289-303.

8. Platzek I. (18)F-Fluorodeoxyglucose PET/MR Imaging in Head and Neck Cancer. PET clinics. 2016;11(4):375-386.

9. Brix $\mathrm{G}$, Lechel $\mathrm{U}$, Glatting $\mathrm{G}$, et al. Radiation exposure of patients undergoing whole-body dual-modality 18F-FDG PET/CT examinations. J Nucl Med. 2005;46(4):608-613. 
10. Chaput A, Robin P, Podeur F, et al. Diagnostic performance of (18) fluorodesoxyglucose positron emission/computed tomography and magnetic resonance imaging in detecting T1-T2 head and neck squamous cell carcinoma. Laryngoscope. 2018;128(2):378-385.

11. Subramaniam RM, Truong M, Peller P, Sakai O, Mercier G. Fluorodeoxyglucosepositron-emission tomography imaging of head and neck squamous cell cancer. AJNR Am J Neuroradiol. 2010;31(4):598-604.

12. Sun R, Tang X, Yang Y, Zhang C. (18)FDG-PET/CT for the detection of regional nodal metastasis in patients with head and neck cancer: a meta-analysis. Oral Oncol. 2015;51(4):314-320.

13. Rohde M, Nielsen AL, Johansen J, et al. Head-to-Head Comparison of Chest XRay/Head and Neck MRI, Chest CT/Head and Neck MRI, and (18)F-FDG PET/CT for Detection of Distant Metastases and Synchronous Cancer in Oral, Pharyngeal, and Laryngeal Cancer. J Nucl Med. 2017;58(12):1919-1924.

14. Zhu L, Wang N. 18F-fluorodeoxyglucose positron emission tomographycomputed tomography as a diagnostic tool in patients with cervical nodal metastases of unknown primary site: a meta-analysis. Surg Oncol. 2013;22(3):190-194.

15. Keraliya AR, Krajewski KM, Braschi-Amirfarzan M, et al. Extracutaneous melanomas: a primer for the radiologist. Insights into imaging. 2015;6(6):707717.

16. Chang AE, Karnell LH, Menck HR. The National Cancer Data Base report on cutaneous and noncutaneous melanoma. a summary of 84,836 cases from the past decade. The American College of Surgeons Commission on Cancer and the American Cancer Society. Cancer. 1998;83(8):1664-1678.

17. O'Regan K, Breen M, Ramaiya N, et al. Metastatic mucosal melanoma: imaging patterns of metastasis and recurrence. Cancer Imaging. 2013;13(4):626-632.

18. Park MJ, Oh JS, Roh JL, et al. 18F-FDG PET/CT Versus Contrast-Enhanced CT for Staging and Prognostic Prediction in Patients With Salivary Gland Carcinomas. Clin Nucl Med. 2017;42(3):e149-e156.

19. Gupta T, Master Z, Kannan S, et al. Diagnostic performance of post-treatment FDG PET or FDG PET/CT imaging in head and neck cancer: a systematic review and meta-analysis. Eur J Nucl Med Mol Imaging. 2011;38(11):2083-2095.

20. Szyszko TA, Cook GJR. PET/CT and PET/MRI in head and neck malignancy. Clin Radiol. 2018;73(1):60-69.

21. Mehanna H, Wong WL, McConkey CC, et al. PET-CT Surveillance versus Neck Dissection in Advanced Head and Neck Cancer. N Engl J Med. 2016;374(15):1444-1454.

22. Lowe VJ, Duan F, Subramaniam RM, et al. Multicenter Trial of [(18)F]fluorodeoxyglucose Positron Emission Tomography/Computed Tomography Staging of Head and Neck Cancer and Negative Predictive Value and Surgical Impact in the N0 Neck: Results From ACRIN 6685. J Clin Oncol. 2019:Jco1801182.

23. Zhang B, Li X, Lu X. Standardized uptake value is of prognostic value for outcome in head and neck squamous cell carcinoma. Acta Otolaryngol. 2010;130(7):756-762. 
24. Wang L, Bai J, Duan P. Prognostic value of 18F-FDG PET/CT functional parameters in patients with head and neck cancer: a meta-analysis. Nucl Med Commun. 2019.

25. Nakamoto $Y$, Tamai $K$, Saga $T$, et al. Clinical value of image fusion from MR and PET in patients with head and neck cancer. Mol Imaging Biol. 2009;11(1):46-53.

26. Huang SH, Chien CY, Lin WC, et al. A comparative study of fused FDG $\mathrm{PET} / \mathrm{MRI}, \mathrm{PET} / \mathrm{CT}, \mathrm{MRI}$, and CT imaging for assessing surrounding tissue invasion of advanced buccal squamous cell carcinoma. Clin Nucl Med. $2011 ; 36(7): 518-525$.

27. Kanda T, Kitajima K, Suenaga $Y$, et al. Value of retrospective image fusion of (1)(8)F-FDG PET and MRI for preoperative staging of head and neck cancer: comparison with PET/CT and contrast-enhanced neck MRI. Eur J Radíol. 2013;82(11):2005-2010.

28. Boss A, Stegger L, Bisdas S, et al. Feasibility of simultaneous PET/MR imaging in the head and upper neck area. Eur Radiol. 2011;21(7):1439-1446.

29. Platzek I, Beuthien-Baumann B, Schneider M, et al.PET/MRI in head and neck cancer: initial experience. Eur J Nucl Med Mol Imaging. 2013;40(1):6-11.

30. Varoquaux A, Rager O, Poncet $A$, et al. Detection and quantification of focal uptake in head and neck tumours: (18)F-FDG PET/MR versus PET/CT. Eur J Nucl Med Mol Imaging. 2014;41(3):462-475.

31. Covello M, Cavaliere C, Aiello M, et al. Simultaneous PET/MR head-neck cancer imaging: Preliminary clinical experience and multiparametric evaluation. Eur $J$ Radiol. 2015;84(7):1269-1276.

32. Kuhn FP, Hullner M, Mader CE, et al. Contrast-enhanced PET/MR imaging versus contrast-enhanced PET/CT in head and neck cancer: how much MR information is needed? J Nucl Med. 2014;55(4):551-558.

33. Platzek I, Beuthien-Baumann B, Schneider M, et al. FDG PET/MR for lymph node staging in head and neck cancer. Eur J Radiol. 2014;83(7):1163-1168.

34. Kubiessa K, Purz S, Gawlitza M, et al. Initial clinical results of simultaneous 18FFDG PET/MRI in comparison to 18F-FDG PET/CT in patients with head and neck cancer. Eur J Nucl Med Mol Imaging. 2014;41(4):639-648.

35. Partovi S, Kohan A, Vercher-Conejero JL, et al. Qualitative and quantitative performance of (1)(8)F-FDG-PET/MRI versus (1)(8)F-FDG-PET/CT in patients with head and neck cancer. AJNR Am J Neuroradiol. 2014;35(10):1970-1975.

36. Cavaliere C, Romeo V, Aiello M, et al. Multiparametric evaluation by simultaneous PET-MRI examination in patients with histologically proven laryngeal cancer. Eur J Radiol. 2017;88:47-55.

37. Becker M, Zbaren P, Casselman JW, Kohler R, Dulguerov P, Becker CD. Neoplastic invasion of laryngeal cartilage: reassessment of criteria for diagnosis at MR imaging. Radiology. 2008;249(2):551-559.

38. Chan SC, Yeh $\mathrm{CH}$, Yen TC, et al. Clinical utility of simultaneous whole-body (18)F-FDG PET/MRI as a single-step imaging modality in the staging of primary nasopharyngeal carcinoma. Eur J Nucl Med Mol Imaging. 2018;45(8):1297-1308.

39. Queiroz MA, Hullner M, Kuhn F, et al. PET/MRI and PET/CT in follow-up of head and neck cancer patients. Eur J Nucl Med Mol Imaging. 2014;41(6):1066-1075. 
40. Schaarschmidt BM, Gomez B, Buchbender C, et al. Is integrated 18F-FDG $\mathrm{PET} / \mathrm{MRI}$ superior to 18F-FDG PET/CT in the differentiation of incidental tracer uptake in the head and neck area? Diagn Interv Radiol. 2017;23(2):127-132.

41. Schaarschmidt BM, Heusch P, Buchbender C, et al. Locoregional tumour evaluation of squamous cell carcinoma in the head and neck area: a comparison between MRI, PET/CT and integrated PET/MRI. Eur J Nucl Med Mol Imaging. 2016;43(1):92-102.

42. Becker $M$, Varoquaux $A D$, Combescure $C$, et al. Local recurrence of squamous cell carcinoma of the head and neck after radio(chemo)therapy: Diagnostic performance of FDG-PET/MRI with diffusion-weighted sequences. Eur Radíol. 2018;28(2):651-663.

43. Queiroz MA, Hullner M, Kuhn F, et al. Use of diffusion-weighted imaging (DWI) in PET/MRI for head and neck cancer evaluation. Eur J Nucl Med Mol/maging. 2014;41(12):2212-2221.

44. Rasmussen $\mathrm{JH}$, Norgaard $\mathrm{M}$, Hansen $\mathrm{AE}$, et al. Feasibility of Multiparametric Imaging with PET/MR in Head and Neck Squamous Cell Carcinoma. J Nucl Med. 2017;58(1):69-74.

45. Ruhlmann V, Ruhlmann M, Bellendorf $A$, et al. Hybrid imaging for detection of carcinoma of unknown primary: A preliminary comparison trial of whole-body PET/MRI versus PET/CT. Eur J Radiol. 2016;85(11):1941-1947.

46. Badger D, Aygun N. Imaging of Perineural Spread in Head and Neck Cancer. Radiol Clin North Am. 2017;55(1):139-149.

47. Sekine T, de Galiza Barbosa F, Kuhn FP, et al. PET+MR versus PET/CT in the initial staging of head and neck cancer, using a trimodality PET/CT+MR system. Clin Imaging. 2017;42:232-239.

48. Abdel Razek AAK, Mukherji SK. State-of-the-Art Imaging of Salivary Gland Tumors. Neuroimaging Clin NAm. 2018;28(2):303-317.

49. Tailor TD, Gupta D, Dalley RW, Keene CD, Anzai Y. Orbital neoplasms in adults: clinical, radiologic, and pathologic review. Radiographics. 2013;33(6):1739-1758.

50. Go JL, Rajamohan AG. Imaging of the Sella and Parasellar Region. Radiol Clin North Am. 2017;55(1):83-101.

51. Akin EA, Torigian DA, Colletti PM, Yoo DC. Optimizing Oncologic FDG-PET/CT Scans to Decrease Radiation Exposure. 2017. https://www.imagewisely.org//media/Image-Wisely/Files/Nuclear-Medicine/Optimizing-Oncologic-FDG-PETCT--Scans-to-Decrease-Radiation-Exposure.pdf?la=en. Accessed 3/25/2019.

52. Kuehl H, Veit P, Rosenbaum SJ, Bockisch A, Antoch G. Can PET/CT replace separate diagnostic CT for cancer imaging? Optimizing CT protocols for imaging cancers of the chest and abdomen. J Nucl Med. 2007;48 Suppl 1:45s-57s.

53. Pfannenberg AC, Aschoff $\mathrm{P}$, Brechtel $\mathrm{K}$, et al. Value of contrast-enhanced multiphase CT in combined PET/CT protocols for oncological imaging. $\mathrm{Br} \mathrm{J}$ Radiol. 2007;80(954):437-445.

54. Pfannenberg AC, Aschoff $P$, Brechtel K, et al. Low dose non-enhanced CT versus standard dose contrast-enhanced CT in combined PET/CT protocols for staging and therapy planning in non-small cell lung cancer. Eur J Nucl Med Mol Imaging. 2007;34(1):36-44. 
55. Tateishi U, Maeda T, Morimoto T, Miyake M, Arai Y, Kim EE. Non-enhanced CT versus contrast-enhanced CT in integrated PET/CT studies for nodal staging of rectal cancer. Eur J Nucl Med Mol Imaging. 2007;34(10):1627-1634.

56. Hall LT, Struck AF, Guglielmo CG, J JC, Wilson MA, Perlman SB. Does the Use of IV Contrast Enhanced CT for Attenuation Correction Affect Clinical Interpretation of Head and Neck PET/CT. The Open Nuclear Medicine Journal. 2011;3:12-18.

57. Rodrigues RS, Bozza FA, Christian PE, et al. Comparison of whole-body $\mathrm{PET} / \mathrm{CT}$, dedicated high-resolution head and neck PET/CT, and contrastenhanced CT in preoperative staging of clinically MO squamous cell carcinoma of the head and neck. J Nucl Med. 2009;50(8):1205-1213.

58. Berthelsen AK, Holm S, Loft A, Klausen TL, Andersen F, Hojgaard L. PET/CT with intravenous contrast can be used for PET attenuation correction in cancer patients. Eur J Nucl Med Mol Imaging. 2005;32(10):1167-1175.

59. Yau YY, Chan WS, Tam YM, et al. Application of intravenous contrast in PET/CT: does it really introduce significant attenuation correction error? J Nucl Med. 2005;46(2):283-291.

60. Ciappuccini R, Aide N, Blanchard D, et al. Incremental Value of a Dedicated Head and Neck Acquisition during 18F-FDG PET/CT in Patients with Differentiated Thyroid Cancer. PLoS One. 2016;11(9):e0162482.

61. Yamamoto Y, Wong TZ, Turkington TG, Hawk TC, Coleman RE. Head and neck cancer: dedicated FDG PET/CT protocol for detection--phantom and initial clinical studies. Radiology. 2007;244(1):263-272.

62. Di Martino E, Nowak B, Krombach GA, et al. [Results of pretherapeutic lymph node diagnosis in head and neck tumors. Clinical value of 18-FDG positron emission tomography (PET)]. Laryngorhinootologie. 2000;79(4):201-206.

63. Bushberg JT. The essential physics of medical imaging. Lippincot Williams and Wilkins; 2011.

64. Berker Y, Franke J, Salomon A, et al. MRI-based attenuation correction for hybrid PET/MRI systems: a 4-class tissue segmentation technique using a combined ultrashort-echo-time/Dixon MRI sequence. J Nucl Med. 2012;53(5):796-804.

65. Boellaard R, Quick HH. Current image acquisition options in PET/MR. Semin Nucl Med. 2015;45(3):192-200.

66. Dickson JC, O'Meara C, Barnes A. A comparison of CT- and MR-based attenuation/correction in neurological PET. Eur J Nucl Med Mol Imaging. 2014;41(6):1176-1189.

67. Bailey DL, Pichler BJ, Guckel B, et al. Combined PET/MRI: Multi-modality Multiparametric Imaging Is Here: Summary Report of the 4th International Workshop on PET/MR Imaging; February 23-27, 2015, Tubingen, Germany. Mol Imaging Biol. 2015;17(5):595-608. 
Figure Captions

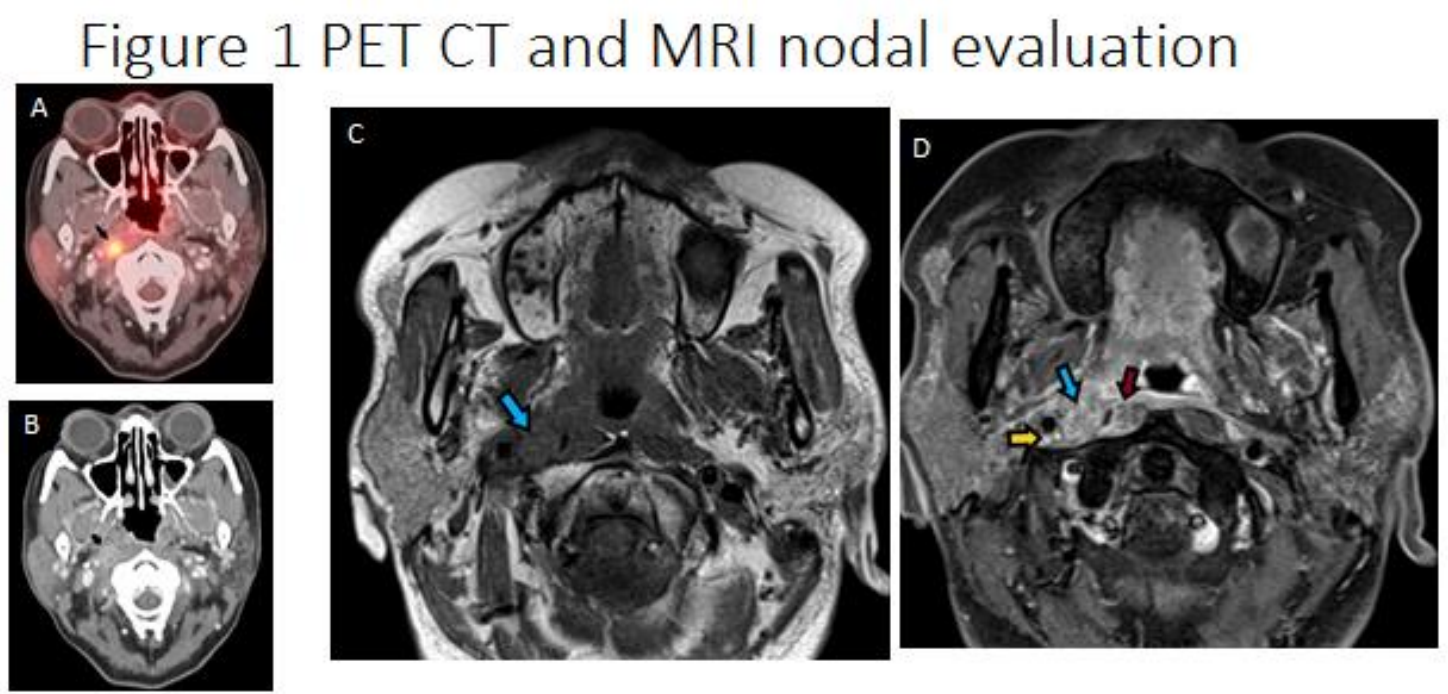

Figure 1: PET/CT and MRI Nodal Evaluation in a patient with recurrent nasopharyngeal carcinoma. Fused axial FDG-PET/CT (A) shows a hypermetabolic right retropharyngeal lymph node (black arrow). Unfused CECT (B) shows corresponding enhancing retropharyngeal lymph node (black arrow). Pre contrast T1-weighted precontrast image (C) shows the metastatic lymph node (blue arrow) to have similar signal intensity to adjacent longus colli muscle. Axial T1-weighted post contrast fat-saturated image (D) shows enhancement of the nodal metastasis (blue arrow), along with enhancement into the adjacent longus colli muscle (red arrow), suggestive of extra nodal extension. There is also enhancement of the soft tissues adjacent to the right internal carotid artery (yellow arrow), compatible with arterial encasement. 
Figure 2: Cancer of Unknown Primary
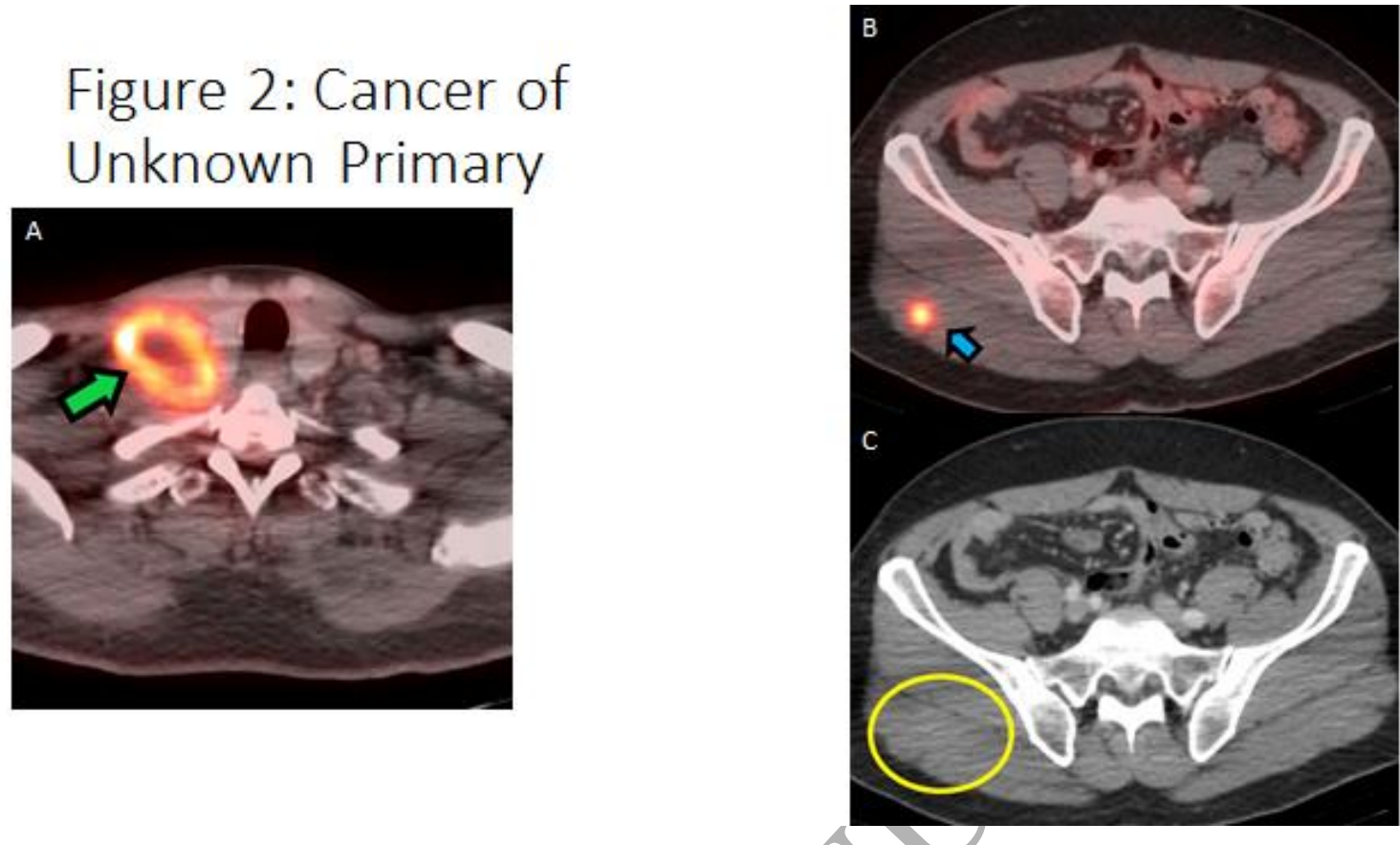

Figure 2: Cancer of Unknown Primary in a patient with palpable right supraclavicular mass. Biopsy showed metastatic squamous cell carcinoma without known primary. Fused axial FDG-PET/CT (A) for staging shows a right supraclavicular mass (green arrow) with peripheral hypermetabolism and central necrosis. The primary site of malignancy was not identified on this PET/CT. Fused axial FDG-PET/CT (B) also shows an additional, small, hypermetabolic metastasis noted in the right gluteus maximus (blue arrow). Unfused axial CECT (C) at the level of the lesion shows the gluteal metastasis is not visible on CT alone (yellow circle). 


\section{Figure 3 Treatment response}
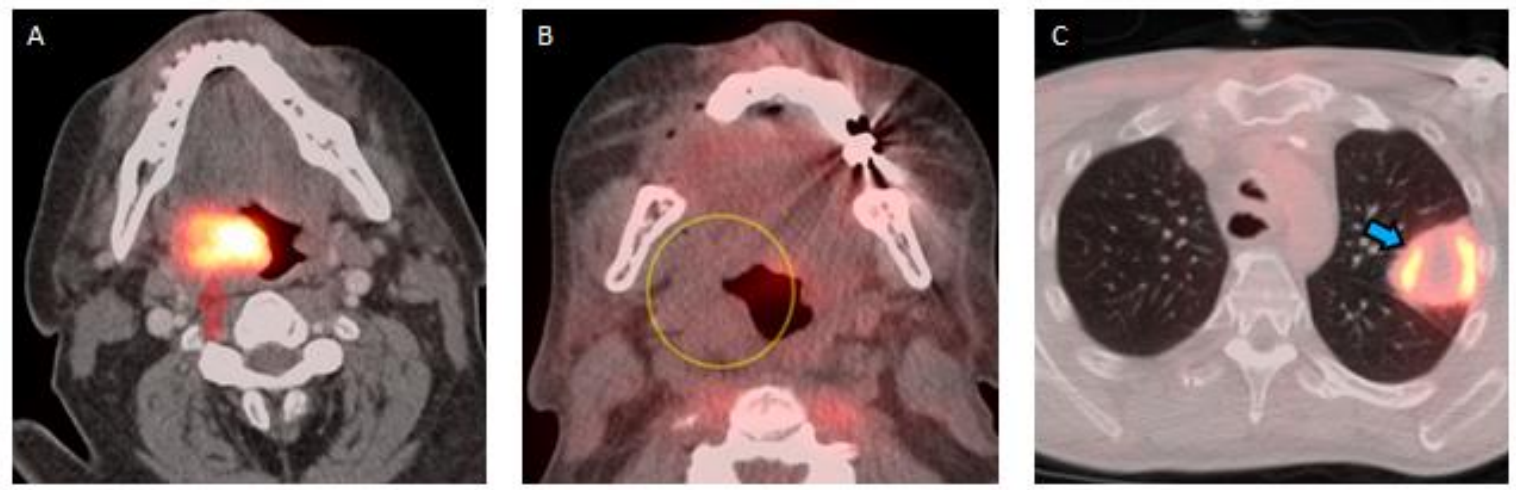

C

Figure 3: Treatment Response with staging and post chemotherapy and radiation treatment FDG-PET/CTs in a patient with T3,N0,M0 Squamous cell carcinoma of the right palatine tonsil and base of tongue. Fused axial FDG-PET/CT (A) for staging shows a hypermetabolic right palatine tonsil and base of tongue mass (red arrow). Fused axial FDG-PET/CT 3 months post completion of chemotherapy and radiation (B) shows no residual hypermetabolic activity in the right tonsillar fossa (yellow circle), compatible complete local response to therapy. Unfortunately, 3 month post therapy fused axial FDG-PET/CT (C) also shows a new, hypermetabolic and partially necrotic left upper lobe mass compatible with distant metastasis and progression of disease (blue arrow). 


\section{Figure 4 PET/MRI Lymph node metastases}

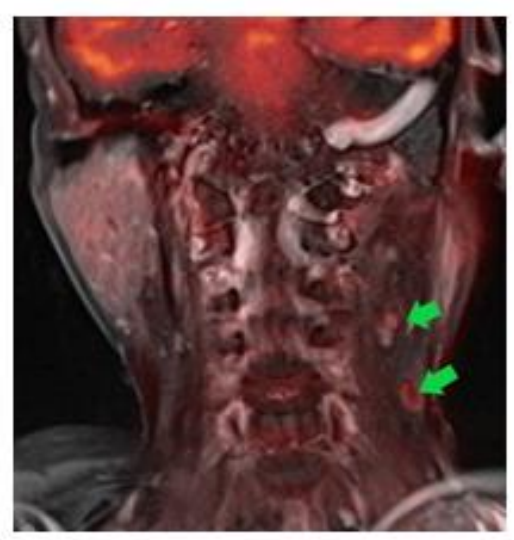

Figure 4: PET/MRI with lymph node metastases in a patient with oropharyngeal squamous cell carcinoma. Fused coronal FDG-PET/MRI with T1-weighted post contrast fat-saturated image shows mildly FDG avid cervical lymph nodes (green arrows) due to nodal metastases. 


\section{Figure 5 Perineural Spread}
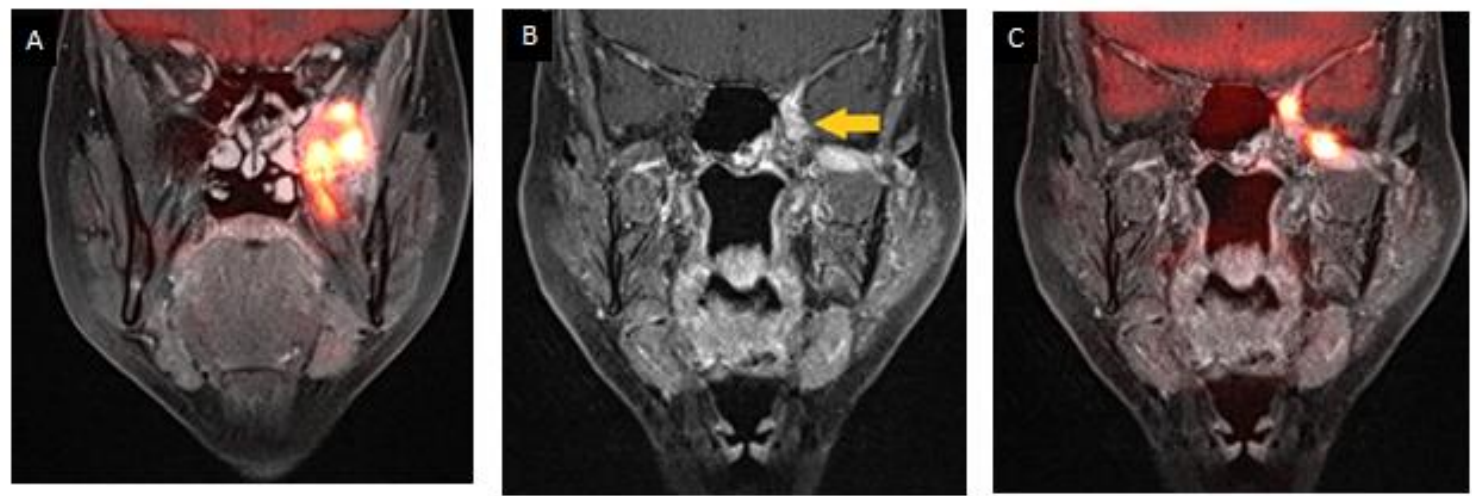

Figure 5: PET/MRI with perineural tumor spread in a patient with squamous cell carcinoma arising in the maxillary sinus. Fused coronal FDG-PET/MRI with T1-weighted post contrast fat-saturated image $(A)$ shows a hypermetabolic, enhancing mass in the left maxillary sinus with local extension. Coronal T1-weighted post contrast fat-saturated image (B) shows soft tissue thickening and enhancement along the superior and inferior orbital fissures (yellow arrow), which is markedly FDG avid on the fused FDG-PET/MRI (C) compatible with perineural tumor spread. 


\section{Figure 6 Dental Artifact}
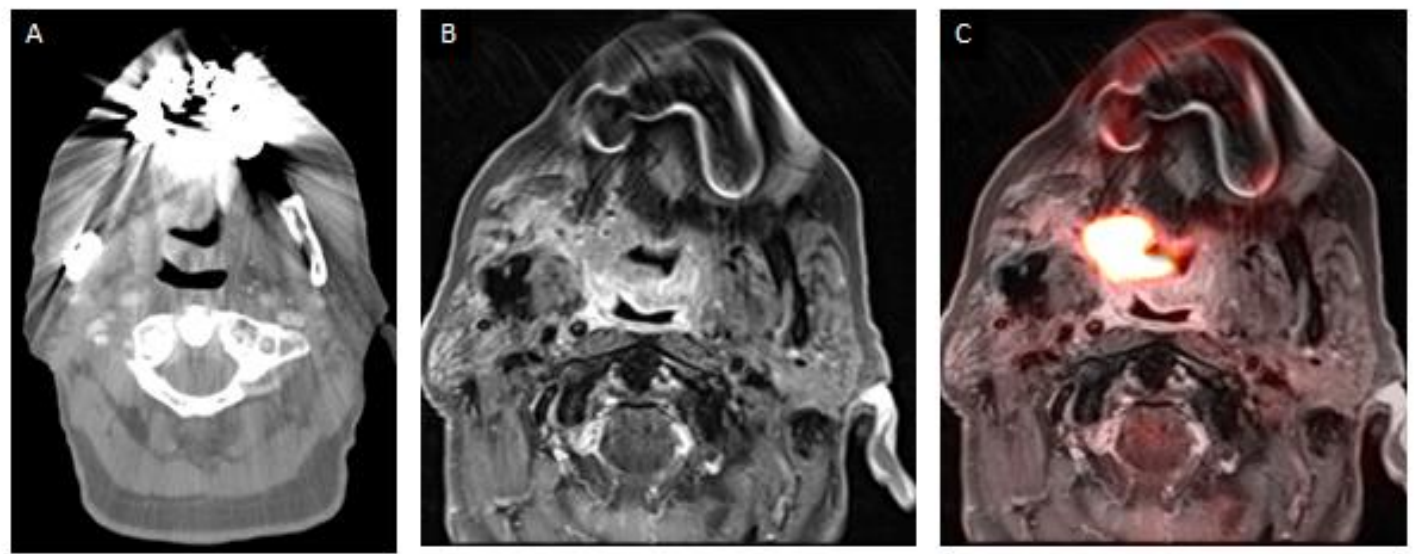

Figure 6: Dental artifact in a patient with suspected recurrent squamous cell carcinoma in the right palatine fossa on clinical exam. Axial CECT (A) shows streak artifact from dental amalgam limiting evaluation of the oropharynx, although there is subtle fullness of the right palatine fossa in the location of clinically suspected recurrence. Axial T1weighted post contrast fat-saturated image (B) obtained during FDG-PET/MRI acquisition shows reduction in metal artifact from dental amalgam compared to CT, allowing the asymmetric soft tissue thickening of the right palatine tonsil to be better appreciated.Fused axial FDG-PET/MRI with T1-weighted post contrast fat-saturated image (C) shows avid FDG uptake in the area of masslike thickening of the right palatine tonsil, compatible with recurrent squamous cell carcinoma in this patient. 

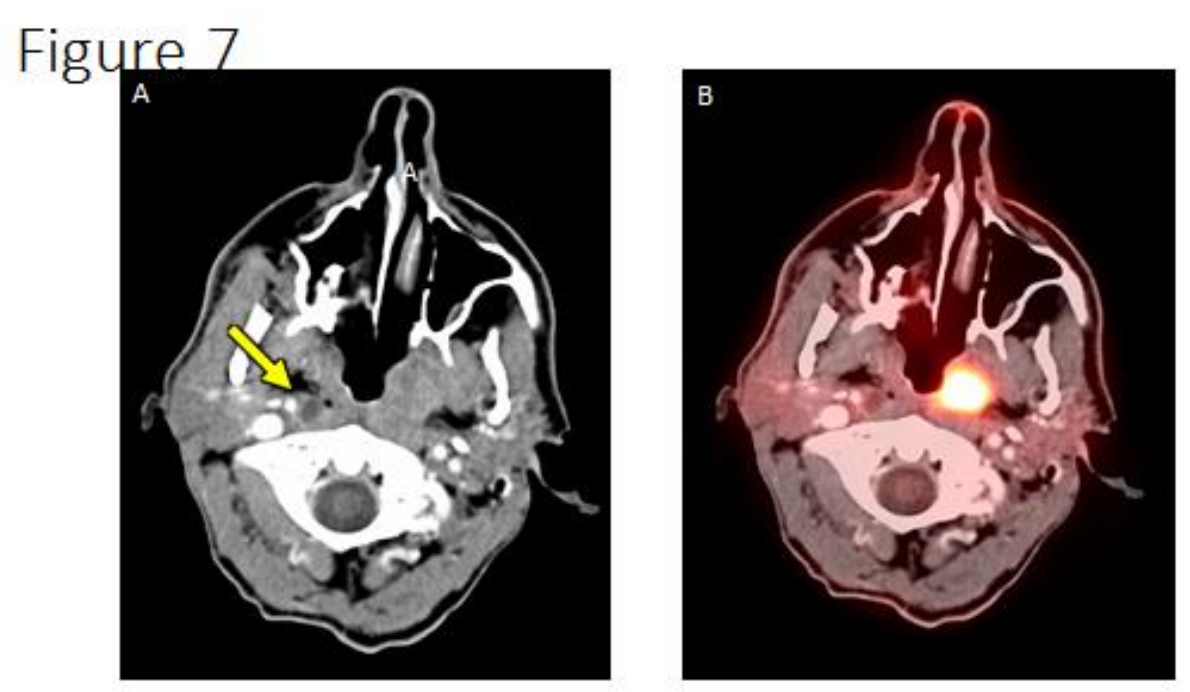

Figure 7: Primary tumor and cystic nodal metastasis evaluation. Axial CECT (A) demonstrates fullness of the left nasopharyngeal mucosa with heterogeneous attenuation. A small, hypodense lesion in the right retropharyngeal space (yellow arrow) is able to be appreciated due to the surrounding, enhancing normal tissues and corresponds to a necrotic retropharyngeal lymph node. Fused axial FDG-PET/CT demonstrates a hypermetabolic left nasopharyngeal mucosa consistent with primary malignancy. The necrotic right retropharyngeal lymph node does not show FDG avidity, but remains suspicious for malignancy given its characteristics on CT. 
Figure 8 a

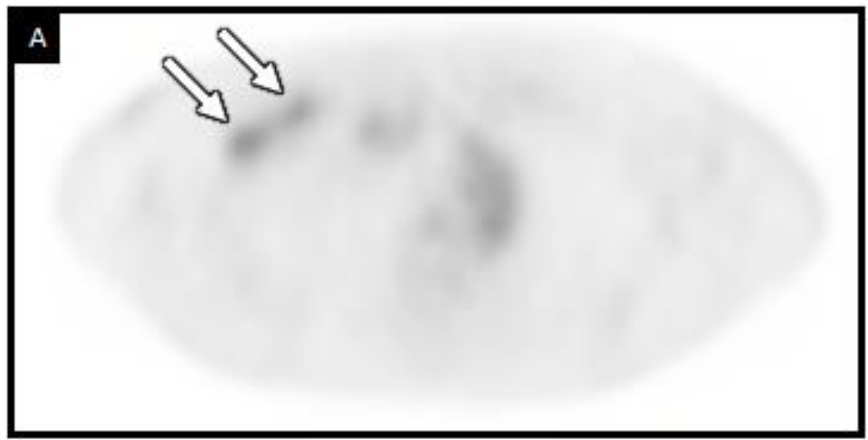

Figure $8 b$

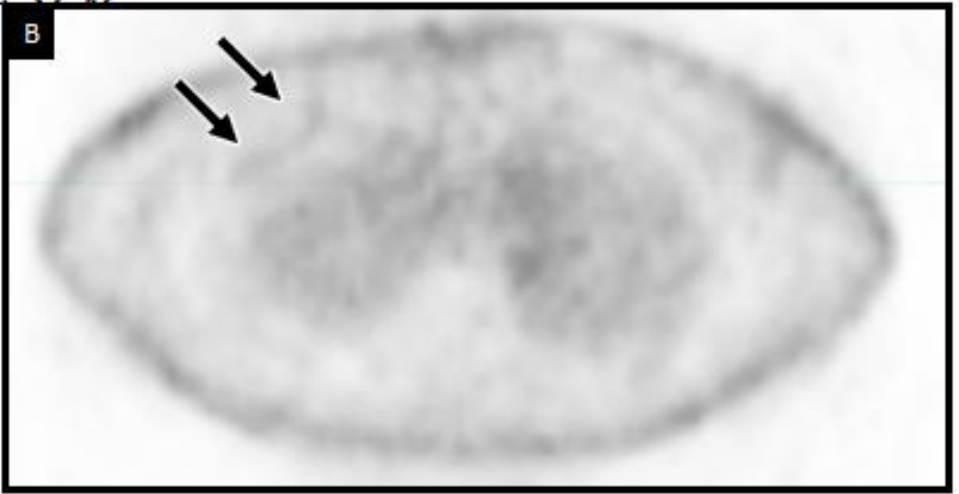




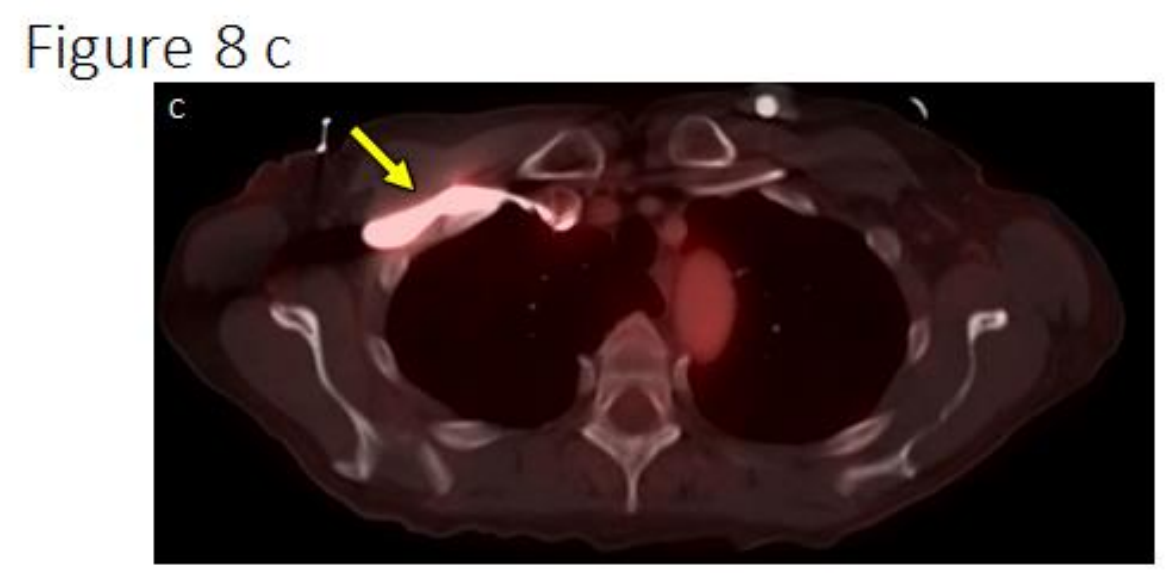

Figure 8: Attenuation Correction. Axial 18F-FDG RET attenuation corrected image (A) demonstrates two foci of abnormal uptake in the right supraclavicular fossa (white arrows). Axial 18F-FDG non-attenuation corrected PET (B) shows minimal uptake in the right supraclavicular fossa on the same slice (black arrows). Review of the fused axial 18F-FDG PET/CT dataset at the same level (C) demonstrates a large bolus of iodinated contrast material in the right supraclavicular fossa (yellow arrow) causing artifactual accumulation of radiotracer activity. 

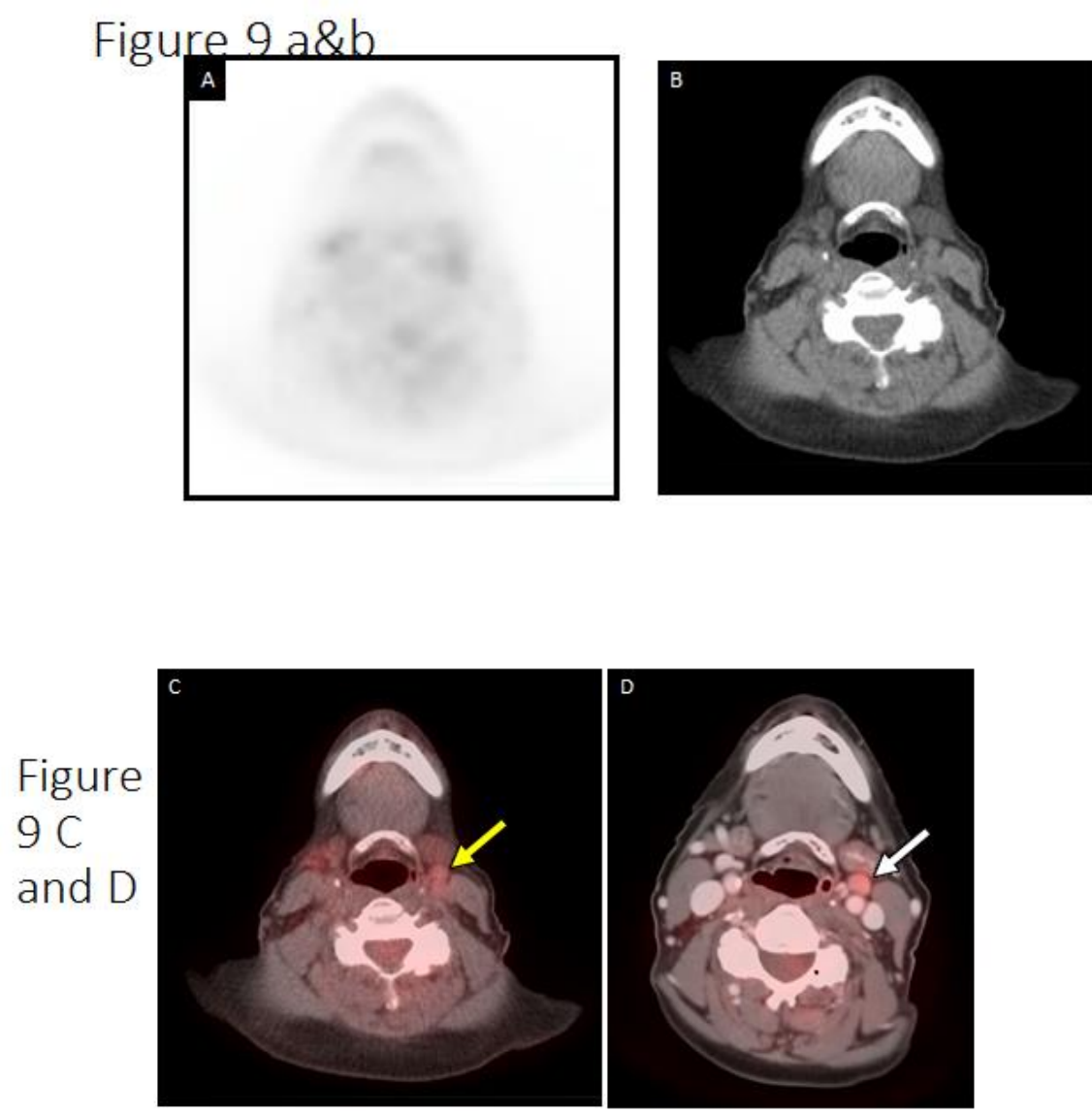

Figure 9: Added value of PET/CT fusion. Axial 18F-FDG PET image (A) through the neck does not demonstrate a definitive focus of abnormal uptake. Axial non-contrast CT through the same level (B) shows no pathologically enlarged lymphadenopathy. Fused axial 18F-FDG PET/CT without contrast (C) shows a small left level 2A lymph node with low uptake (yellow arrow), which is difficult to determine whether it is abnormal metastatic node or a reactive lymph node. Fused axial 18F-FDG PET/CT with iodinated contrast in the same patient more conspicuously demonstrates abnormal, round, mildly hypermetabolic left level 2A lymph node metastasis (white arrow). 


\section{Figure 10}

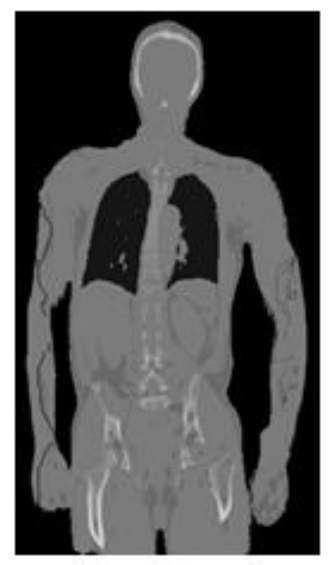

Figure 10: Tissue Segmentation Map. Coronal MR tissue segmentation map derived from DIXON MR sequence and Atlas data. This map is used for attenuation correction of the PET data. Note that the spine, pelvis, and femora are included in the segmentation map, however, the upper extremity bones are not segmented in the software algorithm. 


\section{Figure 11}

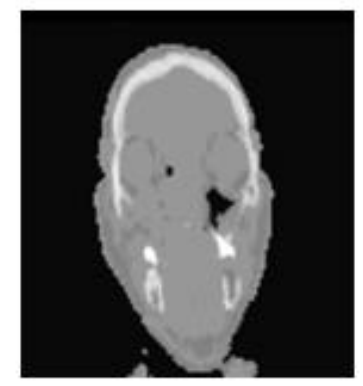

Figure 11: Tissue Segmentation Map of the face with incorrect segmentation. Coronal MR tissue segmentation map of the skull shows portions of the frontal bone, orbits, and paranasal sinuses to be incorrectly segmented as soft tissue. The air-filled paranasal sinuses are also mostly incorrectly segmented. 
Figure 12

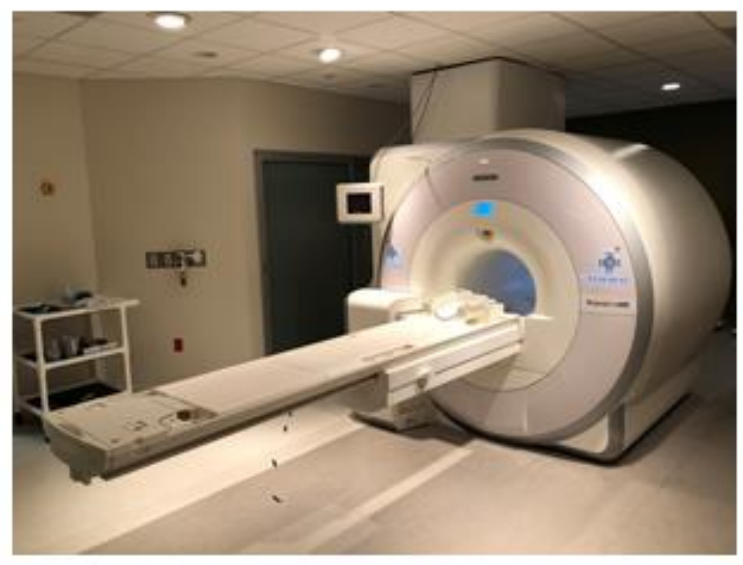

Figure 12: PET/MR Unit. Siemens (Siemens Healthineers AG, Munich, Germany) Biograph MMR PET/MR scanner is shown. The gantry houses an integrated PET detector ring which allows concurrent RET and MR sequence acquisition.

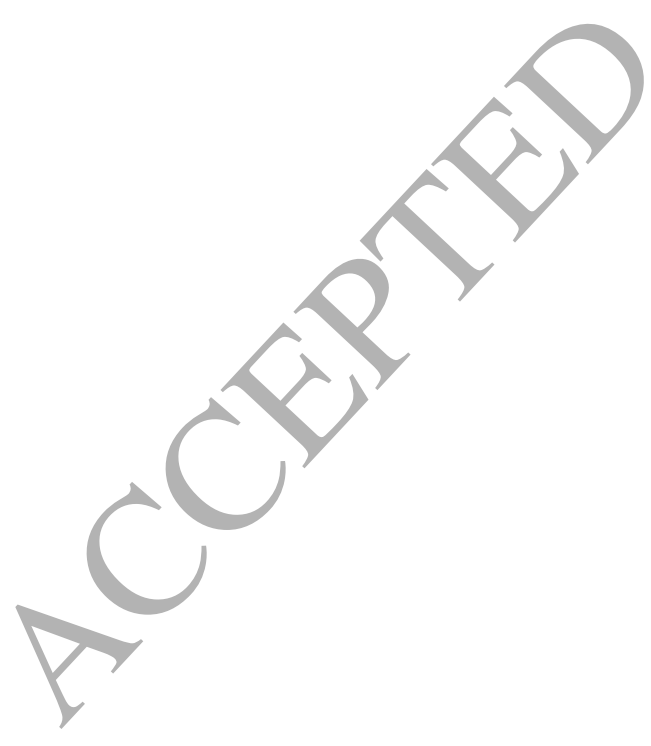




\section{Figure 13}

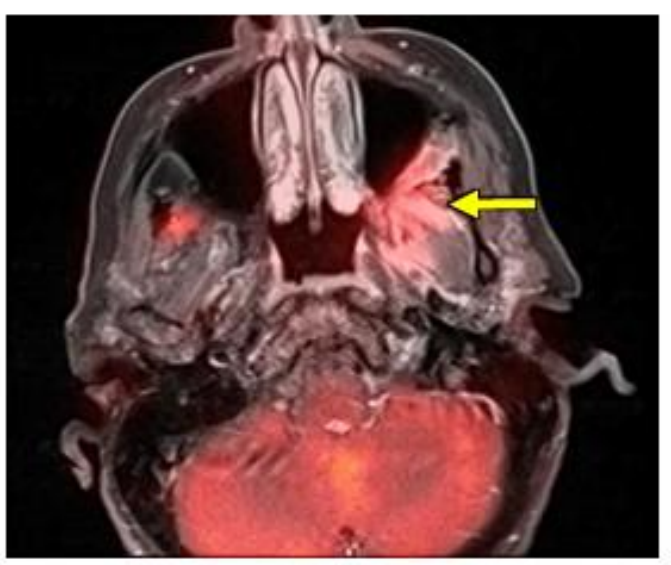

Figure 13: Masticator Space mass. Fused axial PET/MRI with T1-weighted post contrast fat-saturated image demonstrates an avidly-enhancing, FDG avid mass (yellow arrow) of the left infrazygomatic masticator space involving the left medial and lateral pterygoid muscles. 
Figure 14

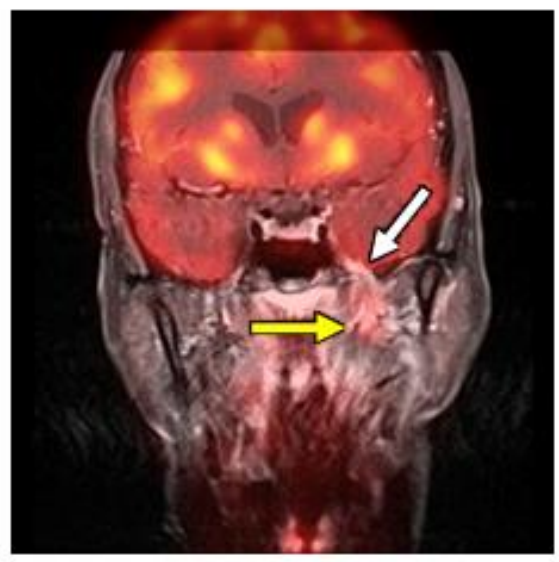

Figure 14: Masticator space mass with perineural spread. Fused coronal PET/MRI with T1-weighted post contrast fat-saturated image shows an infiltrating, FDG avid masticator space mass (yellow arrow) with enhancing, mildly FDG avid perineural tumor spread along the course of cranial nerve V3 (white arrow). 


\section{Figure 15}

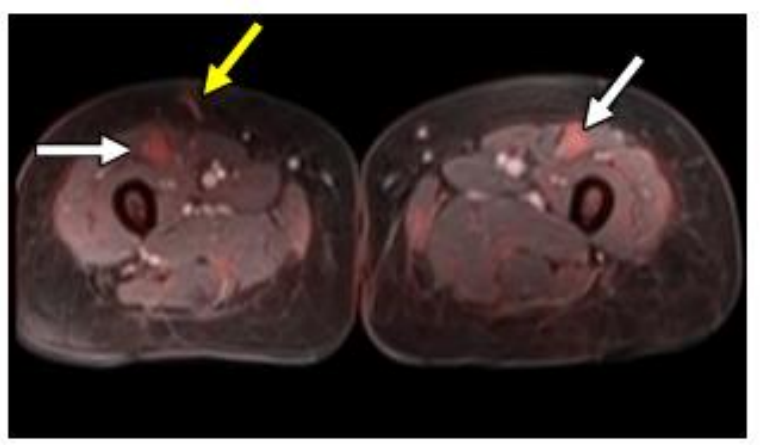

Figure 15: Distant disease. Fused axial PET/MR with T1 VIBE post-contrast fatsaturated image shows enhancing, FDG avid intramuscular (white arrows) and subcutaneous (yellow arrow) nodules suggestive of lymphomatous infiltration.

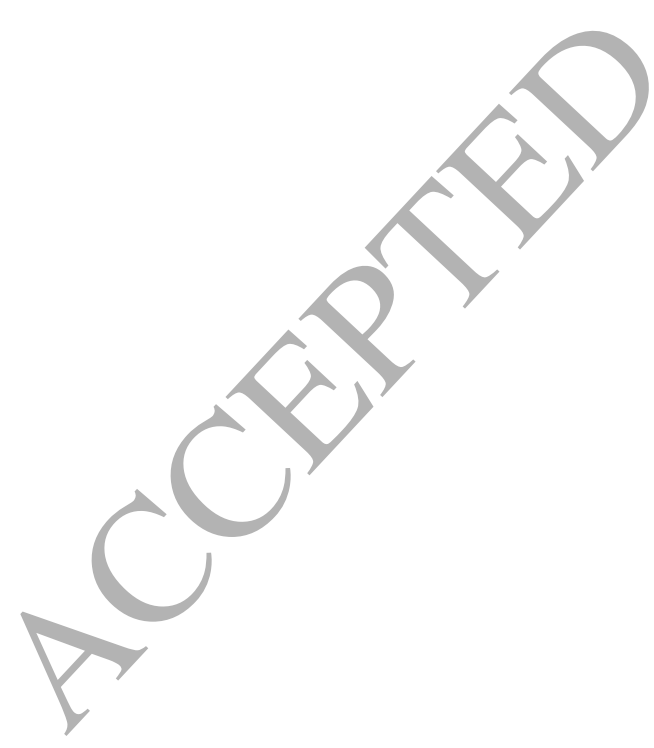


Table 1: Sample oncologic neck soft tissue PET/MRI protocol

\begin{tabular}{|c|c|c|c|c|}
\hline $\begin{array}{l}\text { Region } \\
\text { Scanned }\end{array}$ & MRI Sequence & Plane & Imaging Time & $\begin{array}{l}\text { Concurrent } \\
\text { PET } \\
\text { Acquisition }\end{array}$ \\
\hline $\begin{array}{l}\text { Skull vertex to } \\
\text { mid thighs }\end{array}$ & DIXON & Axial & $\begin{array}{l}13 \text { seconds } \\
\text { (each bed) }\end{array}$ & \multirow{2}{*}{$\begin{array}{l}15 \\
\text { minutes ( } 3 \\
\text { minutes } \\
\text { per bed) }\end{array}$} \\
\hline $\begin{array}{l}\text { Skull vertex to } \\
\text { mid thighs }\end{array}$ & HASTE & Coronal & 2 minutes & \\
\hline Neck & T1 TSE 3mm & Axial & 3 minutes & \multirow{6}{*}{$\begin{array}{l}\text { Continuous } \\
\text { PET } \\
\text { acquisition, } \\
1^{\text {st }} 10 \\
\text { minutes of } \\
\text { imaging } \\
\text { sent to } \\
\text { PACS }\end{array}$} \\
\hline Neck & T1 TSE & Coronal & 2 minutes & \\
\hline Neck & T2 FS TSE & Axial & 3 minutes & \\
\hline Neck & T2 FS TSE & Coronal & 5 minutes & \\
\hline Neck & T1 TSE FS +C & Axial & 5 minutes & \\
\hline Neck & T1 TSE FS +C & Coronal & 3 minutes & \\
\hline $\begin{array}{l}\text { Skull vertex to } \\
\text { mid thighs }\end{array}$ & VIBE & Axial & $\begin{array}{l}17 \text { seconds } \\
\text { (per body } \\
\text { region) }\end{array}$ & None \\
\hline
\end{tabular}


Table 2: Sample oncologic facial PET/MRI protocol

\begin{tabular}{|c|c|c|c|c|}
\hline $\begin{array}{l}\text { Region } \\
\text { Scanned }\end{array}$ & MRI Sequence & Plane & Imaging Time & $\begin{array}{l}\text { Concurrent } \\
\text { PET } \\
\text { Acquisition }\end{array}$ \\
\hline $\begin{array}{l}\text { Skull vertex to } \\
\text { mid thighs }\end{array}$ & DIXON & Axial & $\begin{array}{l}13 \text { seconds } \\
\text { (each bed) }\end{array}$ & \multirow{2}{*}{$\begin{array}{l}15 \\
\text { minutes ( } 3 \\
\text { minutes } \\
\text { per bed) }\end{array}$} \\
\hline $\begin{array}{l}\text { Skull vertex to } \\
\text { mid thighs }\end{array}$ & HASTE & Coronal & 2 minutes & \\
\hline Face & T1 TSE 3mm & Axial & 3 minutes & \multirow{7}{*}{$\begin{array}{l}\text { Continuous } \\
\text { PET } \\
\text { acquisition, } \\
1^{\text {st }} 10 \\
\text { minutes of } \\
\text { imaging } \\
\text { sent to } \\
\text { PACS }\end{array}$} \\
\hline Face & T1 TSE & Coronal & 2 minutes & \\
\hline Face & $\begin{array}{l}\text { T2 FS TSE } 3 \\
\mathrm{~mm}\end{array}$ & Axial & 3 minutes & \\
\hline Face & $\begin{array}{l}\text { T2 FS TSE } 3 \\
\mathrm{~mm}\end{array}$ & Coronal & 5 minutes & \\
\hline Face & T1 TSE FS +C & Axial & 5 minutes & \\
\hline Face & T1 TSE FS +C & Coronal & 3 minutes & \\
\hline Face & DWI & Axial & 2 minutes & \\
\hline $\begin{array}{l}\text { Skull vertex to } \\
\text { mid thighs }\end{array}$ & VIBE & Axial & $\begin{array}{l}17 \text { seconds } \\
\text { (per body } \\
\text { region) }\end{array}$ & None \\
\hline
\end{tabular}


Table 3: Sample oncologic PET/MRI brain protocol

\begin{tabular}{|c|c|c|c|c|}
\hline $\begin{array}{l}\text { Region } \\
\text { Scanned }\end{array}$ & MRI Sequence & Plane & Imaging Time & $\begin{array}{l}\text { Concurrent } \\
\text { PET } \\
\text { Acquisition }\end{array}$ \\
\hline $\begin{array}{l}\text { Skull vertex to } \\
\text { mid thighs }\end{array}$ & DIXON & Axial & $\begin{array}{l}13 \text { seconds } \\
\text { (each bed) }\end{array}$ & \multirow{2}{*}{$\begin{array}{l}15 \\
\text { minutes (3 } \\
\text { minutes } \\
\text { per bed) }\end{array}$} \\
\hline $\begin{array}{l}\text { Skull vertex to } \\
\text { mid thighs }\end{array}$ & HASTE & Coronal & 2 minutes & \\
\hline Brain & DWI & Axial & 2 minutes & \multirow{9}{*}{$\begin{array}{l}\text { Continuous } \\
\text { PET } \\
\text { acquisition, } \\
1^{\text {st }} 10 \\
\text { minutes of } \\
\text { imaging } \\
\text { sent to } \\
\text { PACS }\end{array}$} \\
\hline Brain & T2 FLAIR & Axial & 2 minutes & \\
\hline Brain & T2 FS TSE & Axial & 2 minutes & \\
\hline Brain & T1 MPRAGE & $3 D$ & 5 minutes & \\
\hline Brain & T1 TSE 3mm & Axial & 3 minutes & \\
\hline Brain & T2 FS TSE & Coronal & 3 minutes & \\
\hline Brain & T1 TSE $3 \mathrm{~mm}$ & Coronal & 4 minutes & \\
\hline Brain & T2 SPACE & $3 \mathrm{D}$ & 5 minutes & \\
\hline Brain & T1 MPRAGE +C & $3 D$ & 4 minutes & \\
\hline $\begin{array}{l}\text { Skull vertex to } \\
\text { mid thighs }\end{array}$ & VIBE & Axial & $\begin{array}{l}17 \text { seconds } \\
\text { (per body } \\
\text { region) }\end{array}$ & None \\
\hline
\end{tabular}

